\title{
HS 2237+8154: On the onset of mass transfer or entering the period gap?`
}

\author{
B. T. Gänsicke ${ }^{1,2}$, S. Araujo-Betancor ${ }^{1}$, H.-J. Hagen ${ }^{3}$, E. T. Harlaftis ${ }^{4}$, S. Kitsionas ${ }^{5}$, S. Dreizler ${ }^{6, \star \star}$, and D. Engels ${ }^{3}$ \\ 1 School of Physics and Astronomy, University of Southampton, Southampton SO17 1BJ, UK \\ 2 Department of Physics, University of Warwick, Coventry CV4 7AL, UK \\ ${ }^{3}$ Hamburger Sternwarte, Universität Hamburg, Gojenbergsweg 112, 21029 Hamburg, Germany \\ ${ }^{4}$ Institute of Space Applications and Remote Sensing, National Observatory of Athens, PO Box 20048, Athens 11810, Greece \\ 5 Institute of Astronomy and Astrophysics, National Observatory of Athens, PO Box 20048, Athens 11810, Greece \\ ${ }^{6}$ Universitäts-Sternwarte, Geismarlandstr. 11, 37083 Göttingen, Germany
}

Received 29 October 2003 / Accepted 15 January 2004

\begin{abstract}
We report follow-up observations of a new white dwarf/red dwarf binary HS 2237+8154, identified as a blue variable star from the Hamburg Quasar Survey. Ellipsoidal modulation observed in the $R$ band as well as the $\mathrm{H} \alpha$ radial velocity variations measured from time-resolved spectroscopy determine the orbital period to be $P_{\text {orb }}=178.10 \pm 0.08 \mathrm{~min}$. The optical spectrum of HS $2237+8154$ is well described by a combination of a $T_{\text {eff }}=11500 \pm 1500 \mathrm{~K}$ white dwarf (assuming $\log g=8$ ) and a dM $3.5 \pm 0.5$ secondary star. The distance implied from the flux scaling factors of both stellar components is $d=105 \pm$ $25 \mathrm{pc}$. Combining the constraints obtained from the radial velocity of the secondary and from the ellipsoidal modulation, we derive a binary inclination of $i \simeq 50^{\circ}-70^{\circ}$ and stellar masses of $M_{\mathrm{wd}}=0.47-0.67 M_{\odot}$ and $M_{\mathrm{sec}}=0.2-0.4 M_{\odot}$. All observations imply that the secondary star must be nearly Roche-lobe filling. Consequently, HS 2237+8154 may be either a pre-cataclysmic variable close to the start of mass transfer, or - considering its orbital period - a cataclysmic variable that terminated mass transfer and entered the period gap, or a hibernating nova.
\end{abstract}

Key words. stars: binaries: close - stars: individual: HS 2237+8154 - stars: novae, cataclysmic variables

\section{Introduction}

The currently known population of cataclysmic variables (CVs) comprises more than 1000 systems, with period measurements available for $\simeq 500$ of them (Downes et al. 2001; Kube et al. 2003; Ritter \& Kolb 2003). Compared to these numbers, only very few $\mathrm{CV}$ progenitors have been identified so far. Schreiber \& Gänsicke (2003) have recently analysed 30 wellobserved pre-CVs and found that the sample of known pre$\mathrm{CVs}$ is strongly biased towards systems containing hot (young) white dwarfs and late type (low-mass) companion stars. Testing and improving our understanding of the evolution of pre-CVs

Send offprint requests to: $\mathrm{B}$. T. Gänsicke,

e-mail: Boris.Gaensicke@warwick.ac.uk

* Based in part on observations made at the $1.2 \mathrm{~m}$ telescope, located at Kryoneri Korinthias, and owned by the National Observatory of Athens, Greece, and with the Isaac Newton Telescope, which is operated on the island of La Palma by the Isaac Newton Group in the Spanish Observatorio del Roque de los Muchachos of the Instituto de Astrofisica de Canarias.

$\star \star$ Visiting Astronomer, German-Spanish Astronomical Centre, Calar Alto, operated by the Max-Planck-Institute for Astronomy, Heidelberg, jointly with the Spanish National Commission for Astronomy. through the common envelope phase and of the angular momentum loss mechisms that subsequently brings them into a semidetached configuration will enormously benefit from a larger and unbiased sample of such stars.

We have identified a detached white dwarf/red dwarf binary (Fig. 1) in the course of our studies of the stellar content of the Hamburg Quasar Survey (e.g. Gänsicke et al. 2000; Nogami et al. 2000; Szkody et al. 2001; Gänsicke et al. 2002; Araujo-Betancor et al. 2003). Our follow-up observations show that HS 2237+8154 may belong to the large population of "cold \& old" pre-CVs predicted by Schreiber \& Gänsicke (2003), however, it may as well be a $\mathrm{CV}$ in a peculiar phase of its evolution.

\section{Observations and data reduction}

\subsection{Spectroscopy}

A single identification spectrum of HS 2237+8154 was obtained in June 1991 using the $3.5 \mathrm{~m}$ telescope at Calar Alto equipped with the TWIN spectrograph (Table 1). Gratings with 144 and $160 \AA / \mathrm{mm}$ were used in the blue and red channel respectively providing a resolution of about $6 \AA$. Standard data reduction with flat fielding, bias subtraction as well as 


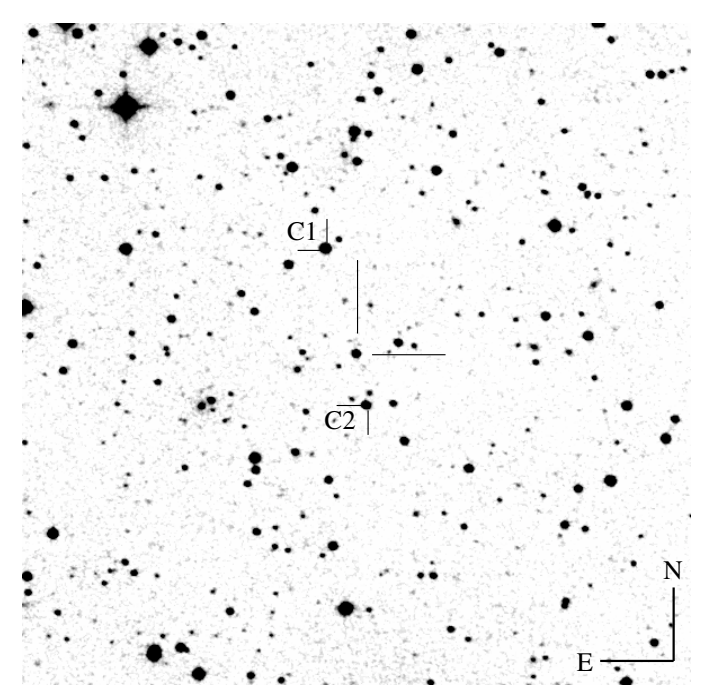

Fig. 1. Finding chart $\left(10^{\prime} \times 10^{\prime}\right)$ for HS $2237+8154$ obtained from the Digitized Sky Survey. The coordinates of the star are $\alpha(2000)=22^{\mathrm{h}} 37^{\mathrm{m}} 15.8^{\mathrm{s}}$ and $\delta(2000)=+82^{\circ} 10^{\prime} 27.5^{\prime \prime}$. The comparison stars used in the analysis of the Kryoneri data are marked "C1" (USNO-A2.0 1650-02587295) and "C2" (USNO-A2.0 1650-02586211).

cosmic-ray filtering was performed in Kiel using IDAS, a long-slit spectroscopy package written by G. Jonas (University Kiel). The TWIN spectrum (Fig. 2) of HS 2237+8154 clearly reveals the composite nature of the object, with prominent $\mathrm{TiO}$ absorption bands in the red part of the spectrum, hallmarks of a late type star, and broad Balmer absorption lines in the blue part of the spectrum, characteristic of the high-gravity atmosphere of a white dwarf. Narrow emission of $\mathrm{H} \alpha$ and $\mathrm{H} \beta$ is detected, which is most likely associated with chromospheric activity on the late-type companion (e.g. Bleach et al. 2000).

Additional time-resolved spectroscopy of HS 2237+8154 was obtained in August 2002 with the Intermediate Dispersion Spectrograph (IDS) on the Isaac Newton Telescope, totalling 30 spectra spread out over one week (Table 1). The IDS was equipped with the R632V grating and the $2048 \times 4100$ pixel EEV10a detector. Using a slit width of $1.5^{\prime \prime}$ the setup provided an unvignetted wavelength range of $\sim 4400 \AA-6800 \AA$ and a spectral resolution of $\sim 2.3 \AA$. Copper-argon wavelength calibrations (arcs) were obtained at the beginning and end of each observation block of HS 2237+8154. The IDS data were bias-subtracted and flat-fielded in a standard manner using the Figaro package within the Starlink software collection. Optimum extraction (Horne 1986) and sky line subtraction was carried out using Tom Marsh's (1989) Pamela package. The dispersion relation was established fitting the arc line positions with a fifth-order polynomial. The rms of the wavelength solution was smaller than $0.1 \AA$ for all spectra. Possible drift of the wavelength-to-pixel scale was accounted for by interpolation between the arcs.

\subsection{Photometry}

We have obtained differential $R$-band photometry of HS 2237+8154 during five nights in September/October 2002
Table 1. Log of the observations.

\begin{tabular}{rcccc}
\hline \hline Date & UT Time & Data & Exp.(s) & Num. Obs \\
\hline Spectroscopy & & & & \\
1991 Jun. 24 & $03: 30-03: 45$ & $R \simeq 6 \AA$ & 900 & 1 \\
2002 Aug. 28 & $00: 32-01: 55$ & $R \simeq 1.6 \AA$ & 600 & 9 \\
2002 Aug. 30 & $01: 35-02: 58$ & $R \simeq 1.6 \AA$ & 600 & 8 \\
2002 Sep. 01 & $01: 51-02: 43$ & $R \simeq 1.6 \AA$ & 600 & 6 \\
2002 Sep. 03 & $23: 35-01: 31$ & $R \simeq 1.6 \AA$ & 600 & 7 \\
Photometry & & & & \\
2002 Sep. 19 & $18: 31-23: 40$ & $R$ & 10 & 961 \\
2002 Sep. 20 & $17: 42-22: 03$ & $R$ & 10 & 960 \\
2002 Oct. 08 & $17: 16-22: 17$ & $R$ & 10 & 982 \\
2002 Oct. 17 & $17: 38-21: 52$ & $R$ & 30 & 415 \\
2002 Oct. 18 & $17: 00-23: 18$ & $R$ & 45 & 417 \\
\hline
\end{tabular}

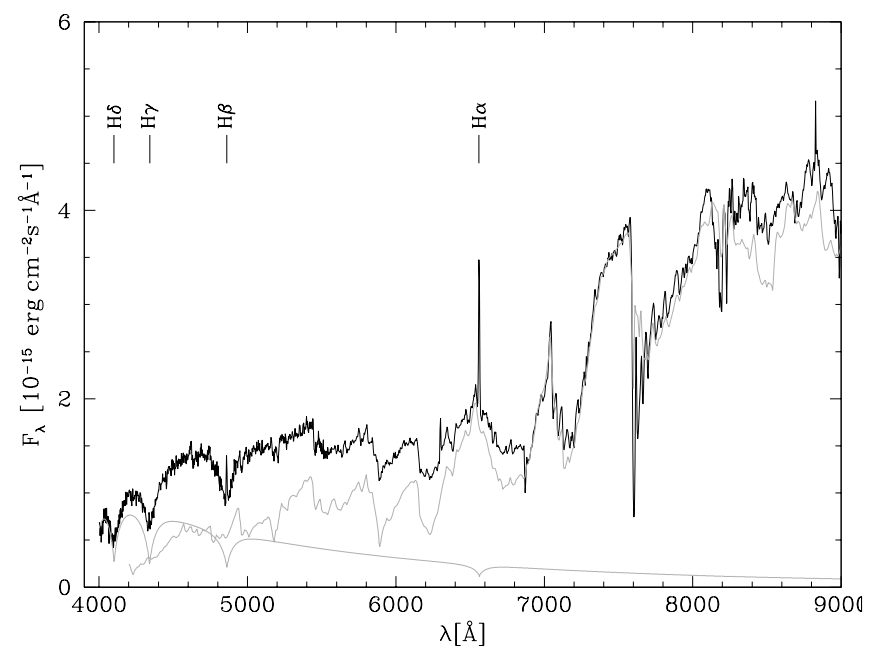

Fig. 2. The Calar Alto TWIN idenfication spectrum of HS 2237+8154. Plotted in gray is a two-component fit consisting of a white dwarf model $\left(T_{\text {eff }}=12000 \mathrm{~K}, \log g=8.0\right)$ and a dM 3.5 template (Gl273).

at the $1.2 \mathrm{~m}$ Kryoneri telescope using a SI-502 $516 \times 516$ CCD camera (Table 1). Bias and dark-current subtraction as well as flat-fielding of all CCD images was carried out in a standard fashion within MIDAS. Because of the relatively poor tracking none of the standard photometry packages could be used to produce light curves from the CCD data without excessive human intervention, and we therefore set up the following reduction pipeline. (1) We extracted detector coordinates and instrumental magnitudes for all objects in each image using the sextractor (Bertin \& Arnouts 1996) in the "classic" aperture photometry mode. In order to account for variable seeing, this step is done as a two-stage process: sextractor is run in a first pass using a relatively large aperture diameter (10 pixel), the median seeing is computed from the resulting object list, and then sextractor is run a second time, using an aperture diameter of 1.5 times the median seeing, which proved to give the best signal-to-noise ratio. (2) One CCD image is designated to be the "reference frame", and the object lists derived from all other images are matched against that of the reference frame. 


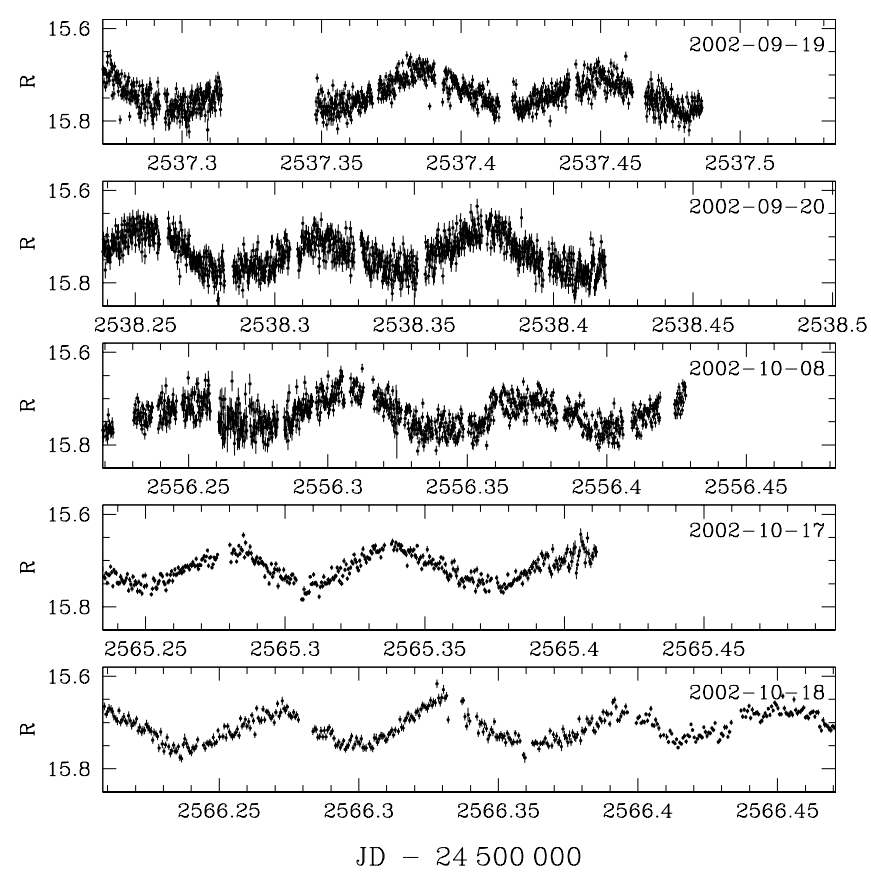

Fig. 3. Differential CCD $R$-band photometry obtained at the Kryoneri $1.2 \mathrm{~m}$ telescope. The $R$ magnitudes have been computed relative to the comparison stars "C1" (09/19 and 09/20) and "C2" (10/08 to 10/18). The data from the first three nights have a lower signal-to-noise ratio because of the relatively short exposure times (10 s).

Considering that most of our CCD images of HS 2237+8154 have only three objects in common, and that no significant distortion/scaling occurs between two different images, we decided to implement the purpose-build matching algorithm smatch. This program computes the distance vectors between all possible pairs of stars in a given image, and compares them with the distances of all possible pairs of stars found in the reference image. A "match" is found if the distance vector between two stars agree within less than a given uncertainty $\epsilon$, with $\epsilon<3$ pixel in the case of the HS $2237+8154$ data. smatch proved to be robust for small numbers of stars (5-6) and provided a better match-rate than more complex codes such as IRAF's xyxymatch. (3) As a consequence of the small CCD detector in use at the Kryoneri telescope (with a field of view of $\left.2.5^{\prime} \times 2.5^{\prime}\right)$ and the poor tracking, special attention has to be given to the choice of the comparison stars, as some of them may move off the detector during the observations. We therefore assign up to four different comparison stars, and compute the differential magnitudes of the target star relative to the brightest comparison visible on a given image.

$R$-band magnitudes of HS $2237+8154$ were derived relative to USNO-A2.0 1650-02587295 $(R=14.5)$ for the observations obtained in September 2002, and relative to USNO-A2.0 1650-02586211 $(R=15.6)$ for the observations obtained in October 2002 (see Fig. 1). During all five nights, HS 2237+8154 was found at a mean magnitude of $R=15.73 \pm$ 0.02 . However, it is important to keep in mind that the systematic error on this $R$ magnitude is of the order $\sim 0.2$ mag due to the limited photometric accuracy of the USNO-A2.0 catalogue (which lists HS $2237+8154$ with $B=16.1$ and $R=15.9$ ).

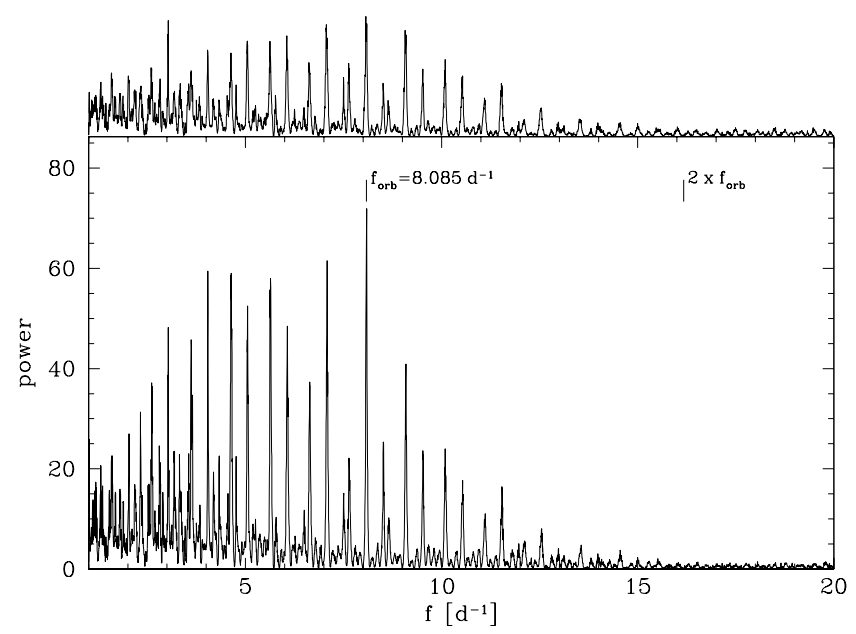

Fig. 4. Main panel: analysis-of-variance periodogram of the radial velocities measured from the $\mathrm{H} \alpha$ emission line. Shown on top is the AOV periodogram of a faked set of radial velocities (see text for details).

For completeness we note that $B=16.1$ has been derived from the HQS observations of HS $2237+8154$.

The light curves of HS 2237+8154 (Fig. 3) display a quasi-sinusoidal variation of the $R$-band magnitude with a peak-to-peak amplitude of $\sim 0.1 \mathrm{mag}$ and a period of $\sim 90 \mathrm{~min}$ during all five nights.

\section{The orbital period of HS $2237+8154$}

\subsection{Radial velocities}

We have measured the radial velocity variation of the narrow $\mathrm{H} \alpha$ emission line in the INT spectra using Gaussian fits. Care was taken to avoid a contamination of the measurement by the broad M-dwarf continuum peak located close to $\mathrm{H} \alpha$. The radial velocities were then subjected to the analysis-of-variance algorithm (AOV Schwarzenberg-Czerny 1989), as implemented in the MIDAS context TSA. The resulting periodogram contains a number of peaks in the range $\sim 2-10 \mathrm{~d}^{-1}$, with the strongest signal at $8.085 \mathrm{~d}^{-1}$ (Fig. 4). We have created a faked set of radial velocities, computing a sine wave with a frequency of $8.085 \mathrm{~d}^{-1}$ and adopting the temporal sampling as defined by the observed spectra. The errors of the observed radial velocity measurements were used to randomly offset the faked radial velocities from the value of the sine wave. The AOV analysis of this faked data set is also shown in Fig. 4. Whereas the spectroscopy strongly suggests an orbital frequency (period) of $8.085 \mathrm{~d}^{-1}$ (178 $\mathrm{min})$, it does not permit a secure identification.

\subsection{Photometry}

The AOV periodogram computed from our photometric data of HS 2237+8154 is shown in Fig. 5. The strongest peak is found at $f \simeq 16 \mathrm{~d}^{-1}(P \simeq 90 \mathrm{~min})$, which corresponds to the period of the quasi-sinusoidal modulation apparent in the light curves (Fig. 3). A second structure in the periodogram is centred at half that frequency, $\simeq 8 \mathrm{~d}^{-1}(P \simeq 180 \mathrm{~min}$ ). Considering that the $R$-band flux of $\mathrm{HS} 2237+8154$ is almost entirely 


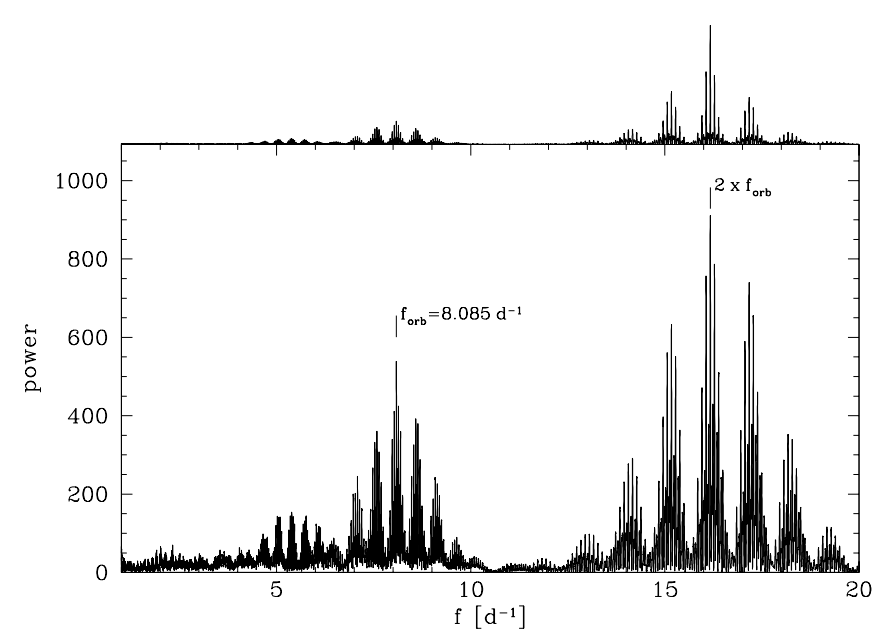

Fig. 5. Main panel: analysis-of-variance periodogram computed from the five nights of differential photometry shown in Fig. 3. On top: the AOV periodogram computed from a sine wave with $f=2 \times f_{\text {orb }}$ reproduces all structures detected in the data.

dominated by emission from the secondary star, the observed quasi-sinusoidal variability is readily explained by either ellipsoidal modulation, in which case $P_{\text {orb }} \simeq 180 \mathrm{~min}$, or by reflection effect of the secondary irradiated by the white dwarf, in which case $P_{\text {orb }} \simeq 90 \mathrm{~min}$. The absence of a signal in the $\simeq 16 \mathrm{~d}^{-1}$ range of the periodogram obtained from the $\mathrm{H} \alpha$ radial velocities (Fig. 4) clearly rules out the second option. We therefore identify the $\sim 8 \mathrm{~d}^{-1}$ signal, which is detected consistently both in the photometric variability as well as in the radial velocity variation, as the orbital period of HS $2237+8154$.

Combining the photometric and spectroscopic observations, we define the following ephemeris

$\phi_{0}=$ HJD $2452514.4112(1)+0.12368(6) \times E$

were phase zero is the time of the blue-to-red crossing of the $\mathrm{H} \alpha$ radial velocities. The most accurate measurement of the orbital period, $P_{\text {orb }}=178.10 \pm 0.08 \mathrm{~min}$, is derived from the photometry which covers a longer time span compared to the spectroscopy.

Figures 6 and 7 show the Kryoneri photometry and the INT radial velocities folded with this ephemeris. The relative phasing of the photometry and spectroscopy is consistent with our interpretation of the photometric variability as ellipsoidal modulation: photometric minima occur during the zerocrossing of the radial velocity curve (i.e. when the front/back side of the secondary star is seen), photometric maxima occur during the radial velocity maxima (i.e. when the secondary star is seen from the side). A trailed spectrogram of the phasefolded IDS spectra around $\mathrm{H} \alpha$ is shown in Fig. 8.

\section{The stellar components in HS $2237+8154$}

\subsection{Spectral fit}

We have fitted the identification spectrum of HS $2237+8154$ (Fig. 2) with a two-component model, consisting of synthetic white dwarf spectra calculated with the code described by Gänsicke et al. (1995), and observed M-dwarf templates from

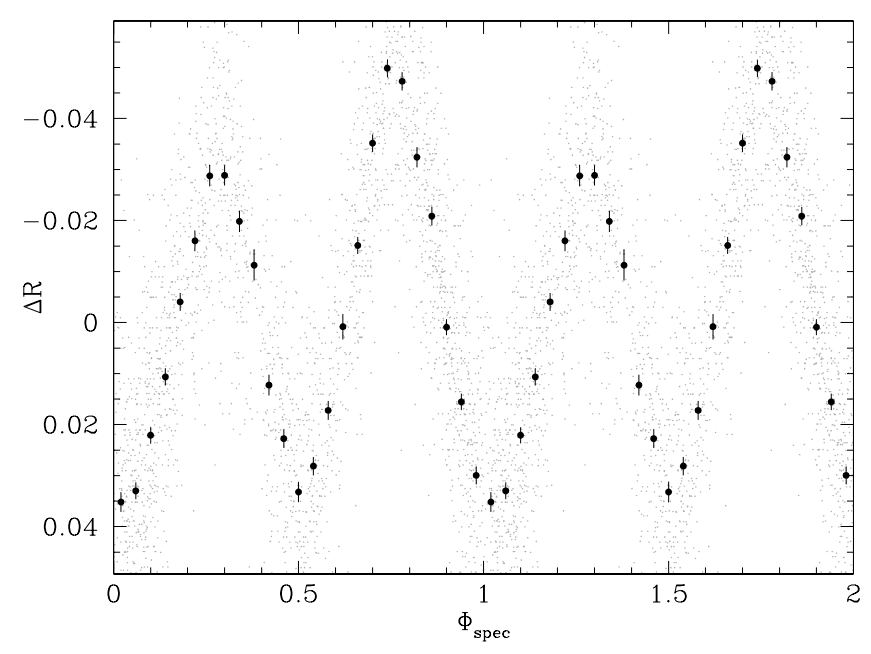

Fig. 6. The Kryoneri photometry average-subtracted and folded using the ephemeris Eq. (1) (gray points) and binned into 25 phase bins (black points). Phase zero is defined as the blue-to-red crossing of the $\mathrm{H} \alpha$ radial velocity (see Fig. 7).

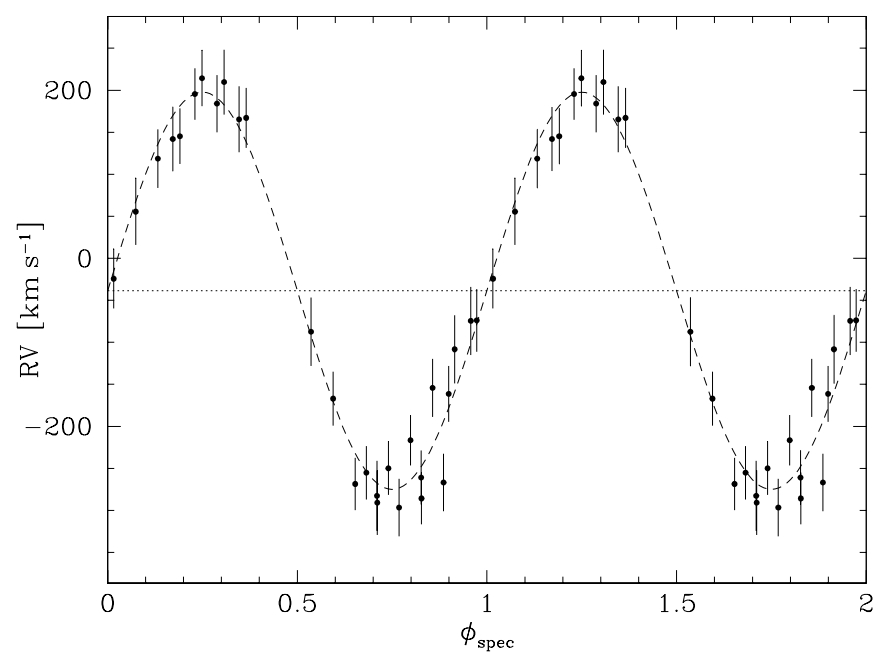

Fig. 7. Radial velocties measured from the $\mathrm{H} \alpha$ emission line, folded over the ephemeris given in Eq. (1). Plotted as dashed line is a sine fit to the radial velocity data which gives a semi-amplitude $K_{\mathrm{sec}}=236 \pm$ $8 \mathrm{~km} \mathrm{~s}^{-1}$ and a systemic velocity $\gamma=-40 \pm 6 \mathrm{~km} \mathrm{~s}^{-1}$.

Beuermann et al. (1998). We fixed the surface gravity of the white dwarf models to $\log g=8$, corresponding to a canonical white dwarf mass of $\sim 0.6 M_{\odot}$, and allowed for temperatures in the range $8000-25000 \mathrm{~K}$. The spectral library of M-dwarfs extended from M 0.5 to M 9. The closest match of the observed spectrum was achieved for a white dwarf with $T_{\mathrm{eff}}=11500 \pm$ $1500 \mathrm{~K}$ and a dM $3.5 \pm 0.5$ companion (Fig. 2). The determination of the secondarys spectral type is relatively robust, as the late-type star completely dominates the emission of the system in the red part of the spectrum. The flux from the white dwarf exceeds that of the companion only for $\lambda \lesssim 5000 \AA$, and consequently its temperature is subject to a relatively large uncertainty. The scaling factors between the model/template spectra and the observed spectrum of HS 2237+8154 allow an estimate of its distance. From the white dwarf (assuming a Hamada-Salpeter (1961) mass-radius relation), 


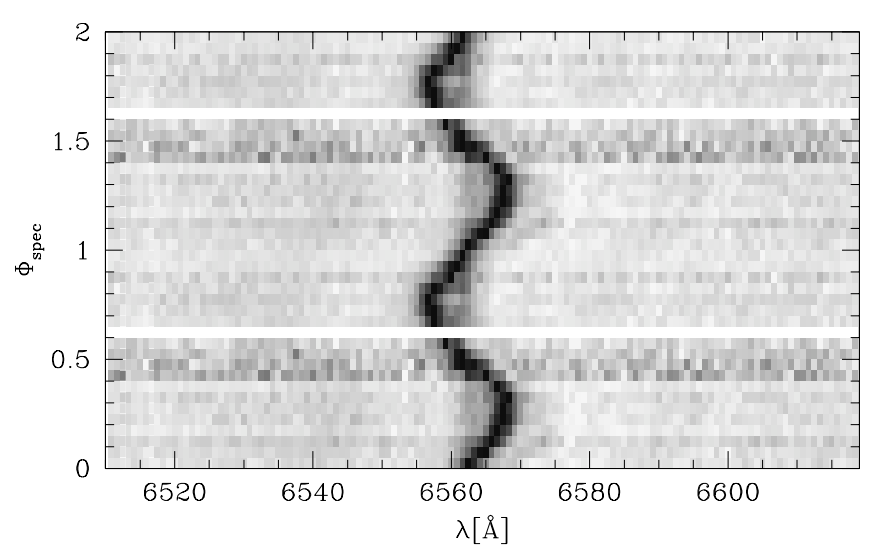

Fig. 8. Trailed spectrogram of the $\mathrm{H} \alpha$ emission line in the IDS spectra of HS $2237+8154$. The 30 individual spectra have been normalised and averaged into 20 phase bins.

we obtained $d=115 \pm 15 \mathrm{pc}$. Obviously, the distance would be lower (higher) if the white dwarf were more (less) massive than $0.6 M_{\odot}$. Assuming that the companion star fills its Roche volume (see the discussion in Sect. 5), the scaling factor of the M-dwarf template implies $d=95 \pm 15$ pc. The agreement between the two values is satisfying, and considering the overall uncertainties involved in the spectral fit $d=105 \pm 25 \mathrm{pc}$ appears to be a conservative estimate, well within the reach of ground-based astrometry programs (e.g. Thorstensen 2003).

\subsection{The radial velocity of the secondary}

Fitting a sine curve to the phase-folded radial velocities (Fig. 7) gives a semi-amplitude $K_{\mathrm{sec}}=236 \pm 8 \mathrm{~km} \mathrm{~s}^{-1}$ and a systemic velocity $\gamma=-40 \pm 6 \mathrm{~km} \mathrm{~s}^{-1}$. Adopting this value of $K_{\mathrm{sec}} \mathrm{im}-$ plies a mass function

$M_{\mathrm{wd}}>f\left(M_{\mathrm{wd}}\right)=\frac{P_{\mathrm{orb}} K_{\mathrm{sec}}}{2 \pi G}=\frac{M_{\mathrm{wd}} \sin ^{3} i}{\left(M_{\mathrm{wd}}+M_{\mathrm{sec}}\right)^{2}}=0.17 M_{\odot}$.

Possible $\left(M_{\mathrm{wd}}, M_{\mathrm{sec}}\right)$ combinations that satisfy this mass function are plotted for a variety of inclinations in Fig. 9. Inclinations $i \gtrsim 75^{\circ}$ are excluded from the absence of eclipses (assuming that the secondary is nearly filling its Roche lobe, see Sect. 5). The typical mass range for field M-dwarfs with a spectral type dM $3.5 \pm 0.5$ is $M_{\mathrm{sec}} \simeq 0.2-0.4 M_{\odot}$ (Kirkpatrick et al. 1991; Baraffe \& Chabrier 1996; Delfosse et al. 1999).

\subsection{Ellipsoidal modulation}

The observed $R$-band light curves show an ellipsoidal modulation with a peak-to-peak amplitude of $\simeq 0.085 \mathrm{mag}$. No evidence for a reflection effect is detected - not too big a suprise considering that the white dwarf is rather cold. Intriguing is the fact that the observed maxima in the $R$-band light curve are not equally bright. A similar effect has been observed in the $R$-band light curve of the pre-CV BPM 71214 (Kawka \& Vennes 2003), who suggested an inhomogenous distribution of starspots as possible explanation. In fact, rapid rotation due to tidal locking may result in enhanced activity on the secondary stars of pre-CVs.

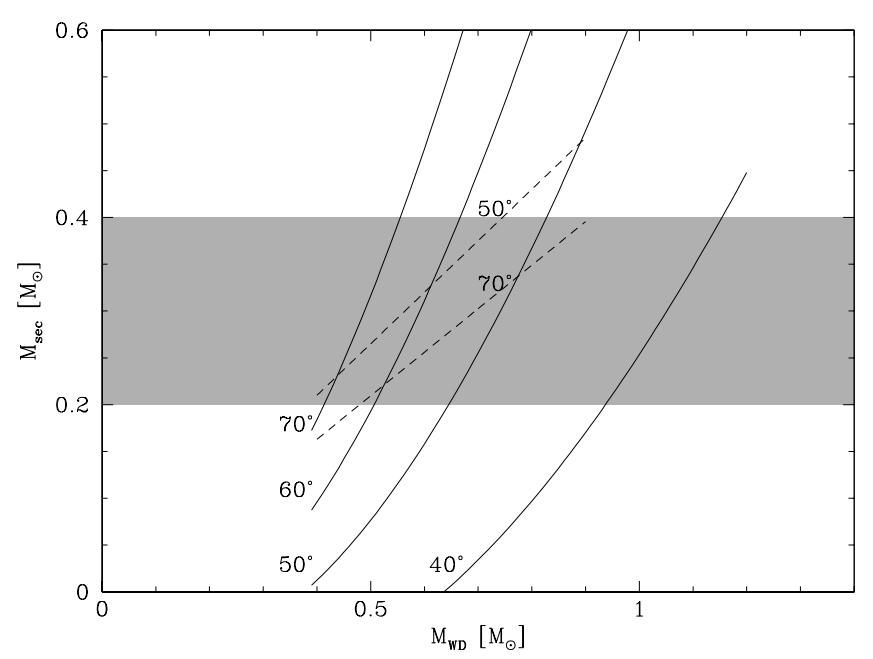

Fig. 9. Constraints on the binary inclination $i$ and the stellar masses, $M_{\mathrm{sec}}$ and $M_{\mathrm{wd}}$. The gray shaded area is the mass range typically found in isolated dM $3.5 \pm 0.5$ stars. The solid lines indicate $\left(M_{\mathrm{wd}}, M_{\mathrm{sec}}, i\right)$ combinations consistent with the measured mass function $f\left(M_{\mathrm{sec}}\right)=$ $0.17 M_{\odot}$. Plotted as dashed lines are constraints derived from modelling the observed ellipsoidal modulation.

We have computed ellipsoidal modulation model light curves for a grid of $\left(M_{\mathrm{wd}}, M_{\mathrm{sec}}, i\right)$ combinations using the analytical formulae of Binnendijk (1974), adopting gravity and limb darkening coefficients of $y=1.38$ and $x=0.80$ (the detailed choice of these two coefficients does not significantly alter the results described below). More specifically, we have searched $\left(M_{\mathrm{wd}}, M_{\mathrm{sec}}\right)$ combinations that reproduce the observed amplitude of the ellipsoidal modulation for a fixed value of $i$. In first order approximation this implies a constant mass ratio $q=M_{\mathrm{sec}} / M_{\mathrm{wd}}$. The resulting constraints on $\left(M_{\mathrm{wd}}, M_{\mathrm{sec}}\right)$ are shown in Fig. 9 for $i=50^{\circ}$ and $i=70^{\circ}$.

Combining the constraints from observed amplitudes of the ellipsoidal modulation and the $\mathrm{H} \alpha$ radial velocity variation provides a lower limit on the Roche-lobe filling factor of the secondary. Assuming the highest inclination and highest mass ratio that are consistent with the mass function $f\left(M_{\mathrm{sec}}\right)$ $\left(i \simeq 70^{\circ}, q=M_{\mathrm{sec}} / M_{\mathrm{wd}} \simeq 0.7\right)$ implies $R_{\mathrm{sec}} \gtrsim 0.75 R_{L_{1}}$ (with $R_{L_{1}}$ the equivalent radius of a Roche-lobe filling secondary). Decreasing $i$ and/or $q$ within the constraints of $f\left(M_{\mathrm{sec}}\right)$ requires $R_{\mathrm{sec}}$ to be closer to $R_{L_{1}}\left(R_{\mathrm{sec}} \gtrsim 0.9 R_{L_{1}}\right.$ for $i \simeq 60^{\circ}$, and $R_{\text {sec }} \simeq R_{L_{1}}$ for $i \simeq 50^{\circ}$ ).

If the secondary star in HS $2237+8154$ has a mass that is typical for its spectral type, and nearly fills its Roche volume, then the combination of all constraints shown in Fig. 9 results in conservative estimates of the binary inclination and of the component masses: $i \simeq 50^{\circ}-70^{\circ}, M_{\mathrm{sec}}=0.2-0.4 M_{\odot}$, and $M_{\mathrm{wd}}=0.47-0.67 M_{\odot}$.

\section{The evolutionary state of HS $2237+8154$}

Based on its composite spectral appearence and orbital period HS $2237+8154$ qualifies as a bona fide pre-CV. However, the spectral type of the secondary is conspicously close to that found in CVs with orbital periods around $3 \mathrm{~h}$ (Beuermann et al. 1998). Could HS $2237+8154$ be a genuine CV caught 
in a state of low accretion? We believe that this is rather unlikely for the following reasons. (1) The system has been found near $B \simeq R \simeq 16$ during six epochs of observations. (2) A number of quiescent dwarf novae are known to reveal the photospheric emission of their stellar components during quiescence. However their optical spectra are always significantly contaminated by the accretion disc, in the form of strong Balmer lines and some more or less noticeable contribution to the continuum (e.g. Szkody et al. 2000). No emission from a quiescent disc is observed in HS $2237+8154$. (3) Many of the strongly magnetic cataclysmic variables (polars) exhibit deep low states during which their spectra are completely dominated by the stellar components (e.g. AM Her Gänsicke et al. 1995; EF Eri Beuermann et al. 2000). The non-detection of Zeemansplitting in the $\mathrm{H} \beta$ to $\mathrm{H} \delta$ absorption lines limits a hypothetical field of the white dwarf in HS 2237+8154 to $B \lesssim 5$ MG, well below the lowest field measured in polars.

Whereas the long-term behaviour and the spectral characteristics argue against a genuine CV nature of HS 2237+8154 the spectral type of its secondary combined with the observed constraints from the observed ellipsoidal modulation and radial velocity variation (Fig. 9) clearly show that the secondary star must be nearly filling its Roche volume.

With a temperature of $T_{\text {eff }}=11500 \mathrm{~K}$ the white dwarf in HS $2237+8154$ is unusually cold compared to the other known pre-CVs and with an implied cooling age of $\sim 400$ million years (for $M_{\mathrm{wd}} \simeq 0.6 M_{\odot}$ ) it is one of the oldest pre-CVs (see Schreiber \& Gänsicke 2003). Taking the available results at face value, we consider three possible evolutionary scenarios for HS 2237+8154. (1) It is a pre-CV close to the onset of mass transfer. Ironically enough, it will be "born" close to the upper edge of the period gap. (2) It is a well-behaved CV - in the context of the disrupted magnetic braking model of $\mathrm{CV}$ evolution - that turned off mass transfer and entered the period gap. (3) It is a CV that is currently hibernating in an extended low state, e.g. as a consequence of a moderately recent nova eruption Shara et al. (1986).

It is interesting to note that two other pre-CVs have recently been suggested to be hibernating novae as they contain nearly Roche-lobe filling secondaries: EC 13471-1258 (O'Donoghue et al. 2003) and BPM 71214 (Kawka \& Vennes 2003). For these two systems, our option (2) is not available, as they have $P_{\text {orb }}=$ $217 \mathrm{~min}$ and $P_{\text {orb }}=290 \mathrm{~min}$, respectively.

\section{Conclusions}

We have identified a detached white/red dwarf binary with an orbital period of $P_{\text {orb }}=178.10 \pm 0.08 \mathrm{~min}$. Our spectroscopic and photometric observations constrain the binary inclination to be $i \simeq 50^{\circ}-70^{\circ}$ and the stellar masses to be $M_{\mathrm{wd}}=0.47-0.67 M_{\odot}$ and $M_{\mathrm{sec}}=0.2-0.4 M_{\odot}$. Modelling the optical spectrum of HS 2237+8154 we find $T_{\text {eff }}=11500 \pm$ $1500 \mathrm{~K}$ for the white dwarf and a spectral type dM $3.5 \pm 0.5$ for the secondary. The distance of the system implied by the flux scaling factors of both stellar components is $d=105 \pm$ $25 \mathrm{pc}$.

The observations strongly suggest that the secondary star in HS 2237+8154 is nearly filling its Roche lobe, and we conclude that the system is either a pre-CV just about to evolve into a semi-detached configuration, a CV that evolved into a detached configuration and entered the period gap, or a hibernating nova. All three possible scenarios are extremely interesting in the context of $\mathrm{CV}$ evolution, and we encourage future work to refine the binary parameter of HS $2237+8154$.

Acknowledgements. We thank Christos Papadimitriou and Dimitris Mislis for carrying out part of the Kryoneri observations. B.T.G. was supported by a PPARC Advanced Fellowship. S.A.B. thanks PPARC for a studentship. We are grateful to Darragh O'Donoghue and to Klaus Beuermann for providing their M-dwarf templates. The HQS was supported by the Deutsche Forschungsgemeinschaft through grants Re 353/11 and Re 353/22. Tom Marsh is thanked for developing and sharing his reduction and analysis packages Pamela and Molly.

\section{References}

Araujo-Betancor, S., Gänsicke, B. T., Hagen, H.-J., Rodriguez-Gil, P., \& Engels, D. 2003, A\&A, 406, 213

Baraffe, I., \& Chabrier, G. 1996, ApJ, 461, L51

Bertin, E., \& Arnouts, S. 1996, A\&AS, 117, 393

Beuermann, K., Baraffe, I., Kolb, U., \& Weichhold, M. 1998, A\&A, 339, 518

Beuermann, K., Wheatley, P., Ramsay, G., Euchner, F., \& Gänsicke, B. T. 2000, A\&A, 354, L49

Binnendijk, L. 1974, Vistas Astron., 16, 61

Bleach, J. N., Wood, J. H., Catalán, M. S., et al. 2000, MNRAS, 312, 70

Delfosse, X., Forveille, T., Mayor, M., Burnet, M., \& Perrier, C. 1999, A\&A, 341, L63

Downes, R. A., Webbink, R. F., Shara, M. M., et al. 2001, PASP, 113, 764

Gänsicke, B. T., Beuermann, K., \& de Martino, D. 1995, A\&A, 303, 127

Gänsicke, B. T., Fried, R. E., Hagen, H.-J., et al. 2000, A\&A, 356, L79

Gänsicke, B. T., Hagen, H. J., \& Engels, D. 2002, in The Physics of Cataclysmic Variables and Related Objects, ed. B. T. Gänsicke,

K. Beuermann, \& K. Reinsch, ASP Conf. Ser., 261, 190

Hamada, T., \& Salpeter, E. E. 1961, ApJ, 134, 683

Horne, K. 1986, PASP, 98, 609

Kawka, A., \& Vennes, S. 2003, AJ, 125, 1444

Kirkpatrick, J. D., Henry, T. J., \& McCarthy, D. W. J. 1991, ApJS, 77, 417

Kube, J., Gänsicke, B. T., Euchner, F., \& Hoffmann, B. 2003, A\&A, 404, 1159

Marsh, T. R. 1989, PASP, 101, 1032

Nogami, D., Engels, D., Gänsicke, B. T., et al. 2000, A\&A, 364, 701

O'Donoghue, D., Koen, C., Kilkenny, D., et al. 2003, MNRAS, 345, 506

Ritter, H., \& Kolb, U. 2003, A\&A, 404, 301

Schreiber, M. R., \& Gänsicke, B. T. 2003, A\&A, 406, 305

Schwarzenberg-Czerny, A. 1989, MNRAS, 241, 153

Shara, M. M., Livio, M., Moffat, A. F. J., \& Orio, M. 1986, ApJ, 311, 163

Szkody, P., Desai, V., Burdullis, T., et al. 2000, ApJ, 540, 983

Szkody, P., Gänsicke, B., Fried, R. E., Heber, U., \& Erb, D. K. 2001, PASP, 113, 1215

Thorstensen, J. R. 2003, AJ, 126, 3017 